\title{
Comparison between Numerical and Experimental for UVP Measurement in Double Bent Pipe with Out-of-Plane Angle
}

\author{
Nobuyoshi Tsuzuki, Treenuson Weerachon, Hiroshige Kikura \\ Research Laboratory for Nuclear Reactors, Tokyo Institute of Technology, Tokyo, Japan \\ Email: ntsuzuki@nr.titech.ac.jp
}

Received 6 July 2014; revised 4 August 2014; accepted 6 October 2014

Copyright (C) 2014 by authors and Scientific Research Publishing Inc.

This work is licensed under the Creative Commons Attribution International License (CC BY). http://creativecommons.org/licenses/by/4.0/

c) (i) Open Access

\section{Abstract}

Twin vortices flow behavior with out-of-plane angle effect in double bent pipe system is studied numerically and experimentally. Double bent pipe system generates very complicated flow behavior including twin vortices in the downstream of the double bent. Moreover, angle from the plane of the double bent forms more complicated flow behavior due to the flow twist by out-of-plane angle. In this study, numerical analysis is examined for this double bent system using three-dimensional CFD code, FLUENT, to reproduce those complicated flow behaviors with twin vortices. Numerical results are compared with experimental results obtained by Ultrasonic Velocity Profiler (UVP). Discrepancy between numerical and experimental result is discussed changing out-ofplane angle, $\alpha$. Velocity profiles obtained by numerical results are converted into UVP profiles, and they are compared with the experimental results by UVP. Consequently, velocity behavior especially around the pipe wall obtained by numerical results is agreed with experimental results.

\section{Keywords}

Double Bent Pipe, Twin Vortices, Vorticity, Comparison, Experimental, Numerical, CFD

\section{Introduction}

Nowadays many engineering plants are utilized in the world. In these plants, materials and energy are supplied to the fabricating machinery, and products are fabricated. To provide materials or remove wastes (including heat), many piping system is utilized because transfer through the piping system is easy to apply since it needs only pipes, and no need to moving machinery for provision. In the case, accurate flowrate measurement is necessary to optimize the efficiency of the plant. 
Flowrate in a pipe is usually monitored using flowmeters. There are a lot of conventional flowmeters, i.e. pressure-drop type, area-type, electromagnetic-type, Coriolis-type etc. Almost all of conventional flowmeters require long inlet length before the measuring point to make fully-developed flow for accurate flowrate measurement. However, the long inlet length demands a lot of space. It is difficult in every facilities to provide such large space, thus, flowrate measurement in real is mainly executed without long enough inlet length. It is very difficult to measure the accurate flowrate when the inlet length is not enough. Therefore, measurement of accurate flowrate without long inlet length became an important issue and many studies are executed with the short inlet length condition. Mattingly et al. [1] studied about velocity profiles near a bent and discussed about the accuracy of flowrate measurement. They applied Laser Doppler Velocimetry (LDV) to obtain the velocity profiles.

In these days, another flow rate measuring method, Ultrasonic Velocity Profiler (UVP) method is focused for accurate flowrate measurement of complicated flow. UVP can measure instantaneous velocity profiles in a line continuously using a transducer from outside of the pipe [2] [3]. Flowrate can be calculated by integration of the velocity profiles obtained by UVP. The flowrate calculation using UVP data for symmetrical straight pipe flow was examined by Takeda [4], and it showed only $0.18 \%$ error using only one line measurement. Furthermore, multiline measurement of UVP provides more accurate calculation of flowrate by reducing the measurement error. Wada et al. [5] studied on the flowrate measurement for asymmetric flow in a straight pipe, changing the number of transducers from one to three, and they concluded the error in the flowrate calculation was only $0.28 \%$ when they apply three measurement lines and circumferential interpolation. There is another flow system which is used for studies about accurate flowrate measurement. That is called double bent pipe system (see Figure 1). In the system, flow was bent twice. The first bent changes flow direction, and immediately the second bent changes the flow direction back to its original flow direction. The double bent generates "twin vortices" [5] [6] in the flow, relatively stable vortices. The twin vortices make the flow characteristics more complicated. The complicated flow behavior, which is difficult to observe by conventional flow meters, was studied experimentally using Particle Image Velocimetry (PIV) [7] [8], and they reported its oscillating flow profiles. However, UVP can observe instantaneous flow velocity profiles. Therefore, Treenuson et al. studied this complicated flow profile with short inlet length using UVP [6] [9]. Three velocity profiles obtained by UVP from different circumferential direction were applied for the calculation of flowrate with circumferential interpolation [9], and they concluded that calculation error is only around 3\% even at the position of $3 D$ ( $D$ : diameter of the pipe) after the double bent. Accordingly, multiline UVP measurement is confirmed as enough accurate method to measure complicated flows such as generated by double bent pipe system.

Understanding the detail of three-dimensional flow behavior from only experimental results is difficult because experimental observation has limitation of measurement resolution. Thus, two- or one-dimensional observation results are accomplished to understand the three dimensional behavior. In fact, one-dimensional UVP observation is applied for the double bent pipe system with many measurements from different positions/angles. However, they are also only parts of the flow behavior. Therefore, numerical calculations are applied to this double bent pipe system to understand the detail of the flow behavior. Accuracy of the calculations will be confirmed by comparison of the velocity profile data with experimental data obtained with UVP.

In this study, numerical analysis using computational fluid dynamics (CFD) code, FLUENT, is examined and detail flow behavior of complicated flow generated by double bent is studied from the numerical data. These numerical analyses execute with fine mesh, and detail three-dimensional velocity profiles are obtained. The numerical results are compared with experimental results obtained by UVP, and the accuracy of the numerical analyses is discussed.

\section{Experimental Results}

\subsection{Theory of UVP Measurement}

UVP uses ultrasound which can transmit into materials including fluid material and also pipe wall material. Figure 2 shows a schematic of UVP measurement. An ultrasonic transducer is placed at the vicinity of a pipe toward the pipe center and emits ultrasound into the pipe. Working fluid in the pipe includes dispersed ultrasound reflecting particles. Emitted ultrasound into the pipe transmits through its measurement line, almost straight, and the ultrasound is reflected by the reflecting particles dispersed in the working fluid. Reflected ultrasound involves information of reflector's motion as Doppler frequency. When the ultrasound reflector moves with the working fluid well, the information of reflector's motion is almost same as the working fluid motion. 


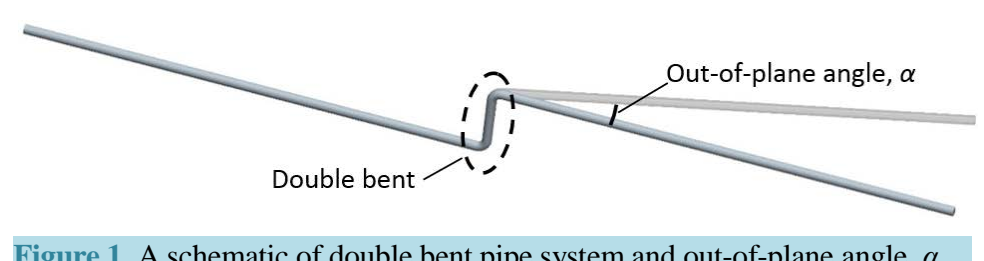

Figure 1. A schematic of double bent pipe system and out-of-plane angle, $\alpha$.

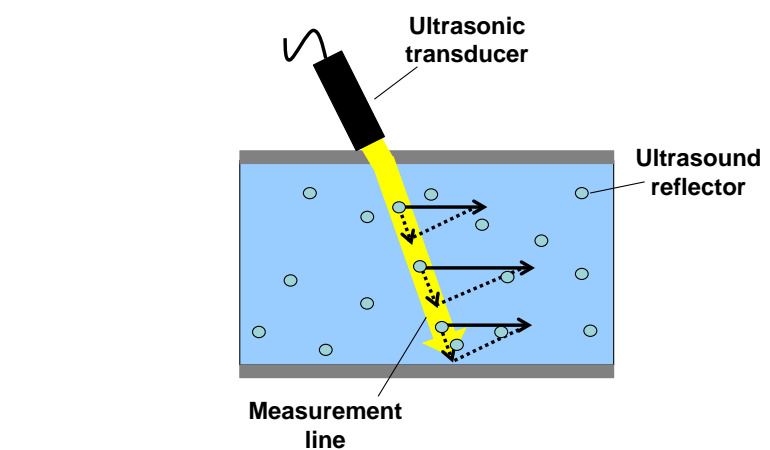

Figure 2. A schematic about UVP measurement.

UVP can extract the Doppler frequency, which include the motion information, in the measurement line by repeating ultrasound emission and reception. Profiles of Doppler frequency can be converted into velocity profiles. Furthermore, sound speed of usual fluid is high enough to measure hundreds times in a second, thus, UVP measurement can monitor instantaneous velocity profile in a line. In fact, ultrasound can transmit through usual pipe wall, and the characteristic allows the ultrasonic transducer to locate out of the fluid, on the wall. In many case, UVP can observe velocity profiles in the pipe from outside of a pipe without any installation.

The detail of UVP is also described in [10].

UVP can measure Doppler frequency profiles, that is, the information obtained by UVP is about velocity component along the measurement line, forward and backward speed from the transducer. Usually, velocity profiles are calculated by assuming main flow direction. It is clear that the main flow direction is almost same as pipe direction in the case of pipe flow.

\subsection{Experimental Results Obtained by UVP}

Experimental measurements have been executed using the double elbow system [7], which generates complicated flow behavior of twin vortices, described above. The schematic figures of the apparatus are shown in Figures 3-4.

A water pump generates circulating flow, and the flowrate is adjusted by a bypass valve (bypass line). The main flow is monitored by an electro-magnetic flowmeter. The flow profile is at first uniformed by a flow conditioner, and enough developed horizontal flow comes to around the double bent. Two bents of $90^{\circ}$ make complicated flow, and the velocity profiles at the downstream of the double bent were measured after $3 \mathrm{D}, 4 \mathrm{D}, 5 \mathrm{D}$ after the double bent exit. The flow goes back to the water tank and circulated again.

At the test section, UVP measurements were executed from all the circumferential direction of every $10^{\circ}$, toward the pipe center. The ultrasonic transducer for UVP is also inclined toward the pipe direction as incident angle of $10^{\circ}$ as shown Figure 3 in every measurement. That can allow measuring also the velocity component along the pipe direction, i.e. the main flow direction.

The effect of out-of-plane angle was also studied during the experiment. Definition of out-of-plane angle is the same as already shown in Figure 1. When the out-of-plane angle exists, the twin vortices are twisted and more complicated flow appears. The accuracy of flowrate calculation for this complicated flow was revealed using UVP measurement in this relating study.

\subsection{Summary of Experimental Results}

Experimental confirmation of flowrate calculation assuming all the flow direction is the same as the pipe direc- 
tion was examined [5] [6]. Figure 5 depicts an example of time-averaged velocity profile for out-of-plane angle $\alpha=0^{\circ}$ and circumferential angle, $\phi=0^{\circ}$. Circumferential angle, $\phi$, is defined same as former study [6], which is explained in Figure 6. Horizontal axis means normalized position by the pipe radius and vertical axis means velocity at the position. Error bar indicates the standard deviation of the measurement. 2000 velocity profiles are averaged and each profile was obtained every $19 \mathrm{~ms}$. Velocity profiles are oscillating, however, the flowrate calculated by the interpolation of the profiles indicates that the error of the flowrate measurement is only $3 \%$ [6]. Figures 7(a)-(d) depict summaries of normalized radial velocity profiles with out-of-plane angle, $\alpha=16^{\circ}$, at $3 D$ after the double elbow. Integration of these profiles provide the flowrate and it is concluded that the error of this flowrate calculation for this complicated flow is lower than $5 \%$ in many cases, as shown in Table 1. Other data for the distance from double elbow, $3 D, 4 D$ and $5 D$; the out-of-plane angle $\alpha=0$ and $16^{\circ}$ are obtained experi-

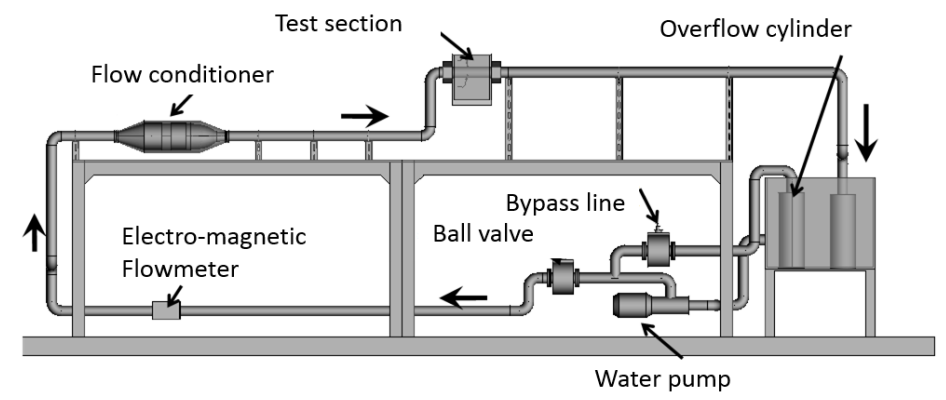

Figure 3. Experimental apparatus of double bent pipe flow.

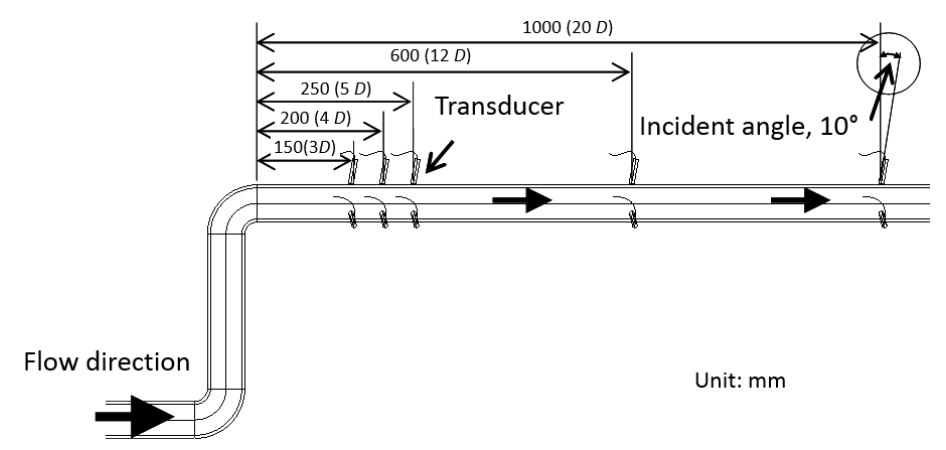

Figure 4. Configuration around the test section and measurement points (2 D - 20 D).

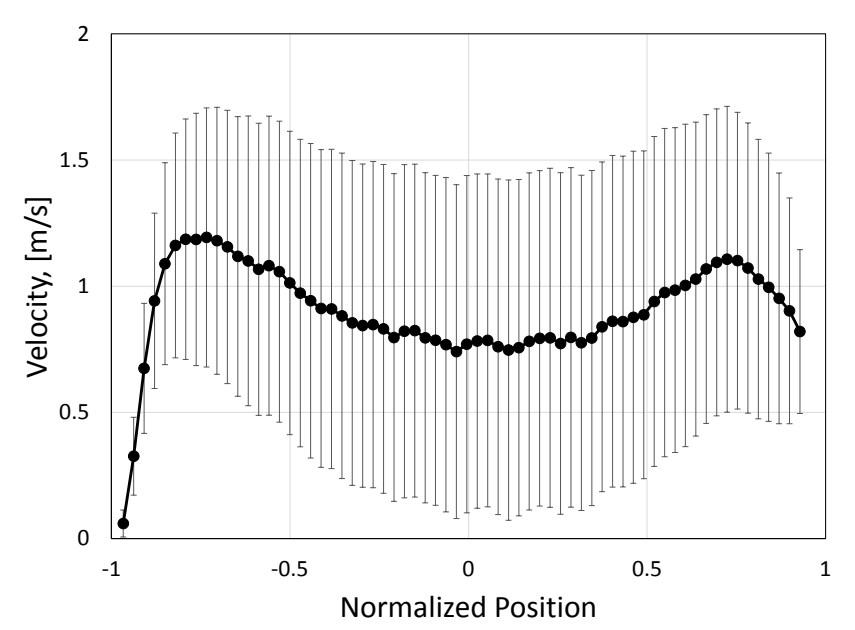

Figure 5 . An example of velocity profile obtained by UVP at $3 D$ after the double elbow, $\alpha=16^{\circ}, \phi=0^{\circ}$. 


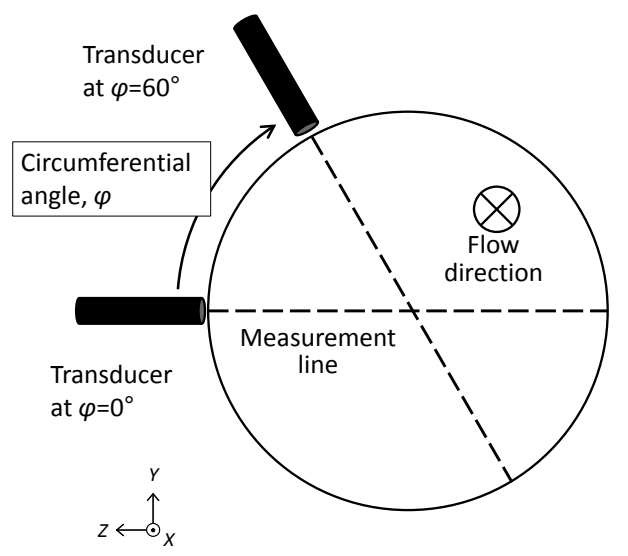

Figure 6. Circumferential angle $\phi$.

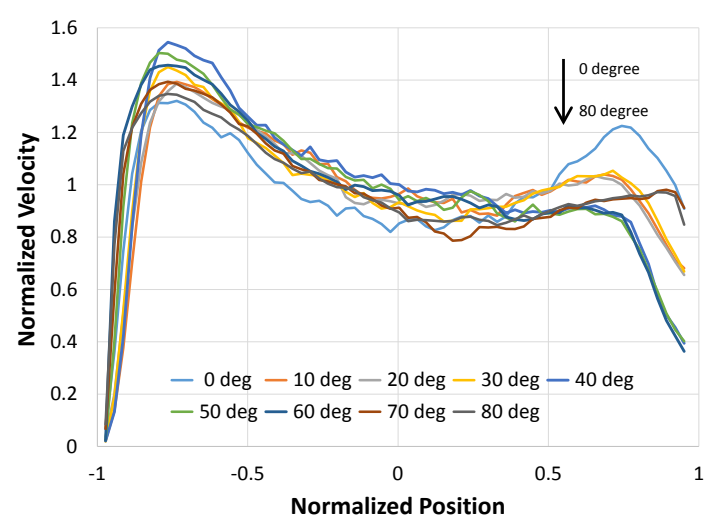

(a)

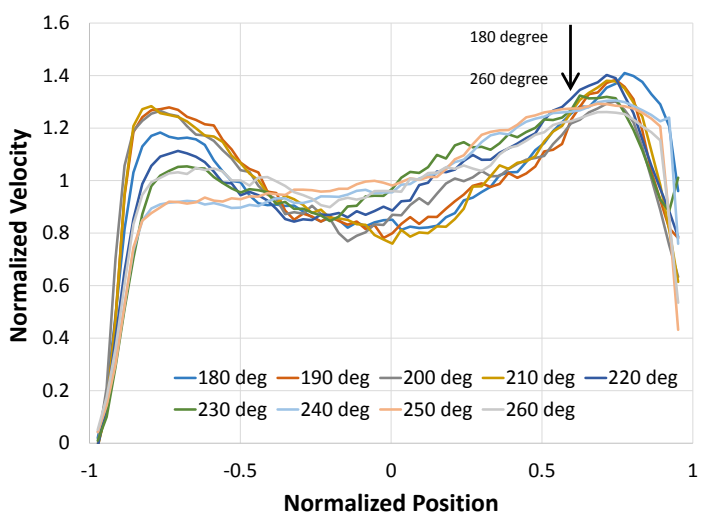

(c)

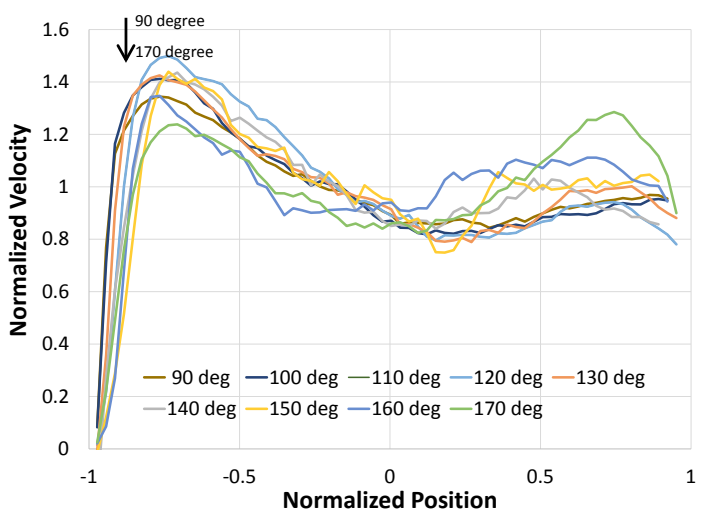

(b)

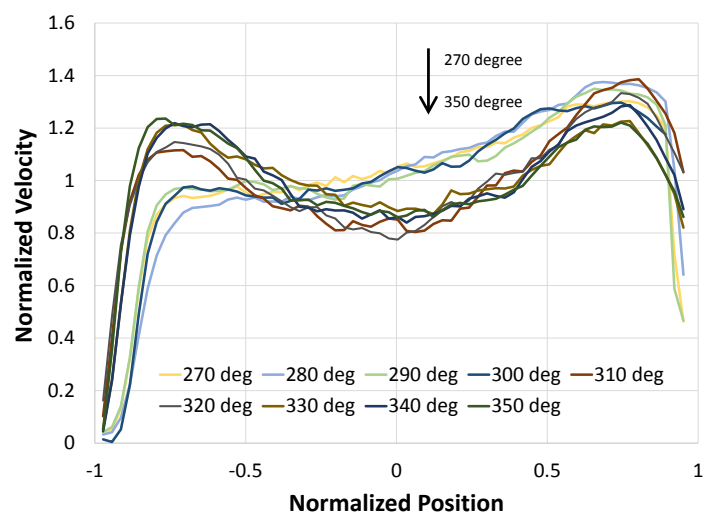

(d)

Figure 7. Normalized radial velocity profiles of every $10^{\circ}$ circumferential angle, $\phi$, obtained by UVP experimentally at $3 D$ after the double elbow, $\alpha=16^{\circ}$. (a) $\phi=0^{\circ}-80^{\circ}$; (b) $\phi=90^{\circ}-170^{\circ}$; (c) $\phi=180^{\circ}-260^{\circ}$; (d) $\phi=270^{\circ}-350^{\circ}$.

Table 1. Error of the flowrate calculation from UVP results ([X]: used number of transducers).

\begin{tabular}{cccc}
\hline$\alpha$ & $3 D$ & $4 D$ & $5 D$ \\
\hline 0 & $3.08 \%[5]$ & $1.60 \%[5]$ & $0.86 \%[4]$ \\
16 & $3.50 \%[6]$ & $-0.18 \%[5]$ & $4.58 \%[5]$ \\
\hline
\end{tabular}

mentally by UVP measurement. These detail profiles are all shown in [6]. 


\section{Numerical Result}

\subsection{Calculation Model}

The condition of the relating experimental results described above is reproduced in numerical condition for a 3-D CFD code, FLUENT.

The calculation configuration for the double bent pipe system was fabricated considering the experimental apparatus, Figure 1.

Enough long length (=20 D, D: diameter of the pipe, $50 \mathrm{~mm}$ ) is placed before the double bent. Two $90^{\circ}$ bents are assigned at both ends of vertical straight pipe (length: $5 \mathrm{D}$ ). Flow goes up in the straight pipe and flow is bent back to horizontal. The horizontal pipe length after the double bent has also enough length (=40 $D$ ). Flow behavior is obtained after the double bent at 3, 4, and $5 \mathrm{D}$ after the exit of double bent. The out-of-plane angle, $\alpha$, is varied among $0^{\circ}, 8^{\circ}$ and $16^{\circ}$ as same as the experimental setup.

Example of mesh distribution around the double bent is depicted in Figure 8.

In former study [6], typical mesh size was set as $0.8 \mathrm{~mm}$, and the flow profiles were studied. In this study, typical mesh size of cross-section is set as $0.1 \mathrm{~mm}$ to make enough detail contour plots for understanding flow behavior, and much more fine mesh is placed at the vicinity of the wall (see Figure 8). Another mesh size along the pipe is changed from $0.5 \mathrm{~mm}$ to $5 \mathrm{~mm}$, considering complexity of the flow inside. Mesh distribution around the double bent is shown in Figure 9. Total number of meshes is approximately 2.3 million.

The CFD code, FLUENT, is applied to calculate in this study as described above. The turbulence model used is RNG k- $\varepsilon$ model considering convergence of each model and its calculation cost (memory and calculation time). Enhanced wall treatment is also used as a wall function. Due to fine meshes, PISO scheme is necessary as coupling scheme between velocity and pressure, and PRESTO! Scheme is used for spatial discretization of pressure, and QUICK scheme is applied for other spatial discretization.

Boundary condition of inlet is given as velocity inlet and the velocity is set to reproduce the experimental condition, Reynolds number $R e \approx 40,000$. The boundary condition of outlet is pressure outlet. Thermal analysis is not included in this study, and physical properties of the working fluid are determined as those of water at room temperature $\left(20^{\circ} \mathrm{C}\right)[11]$.

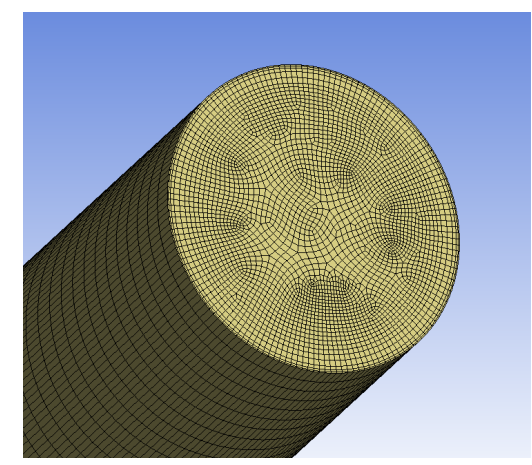

Figure 8. Mesh distribution at cross-section of the pipe.

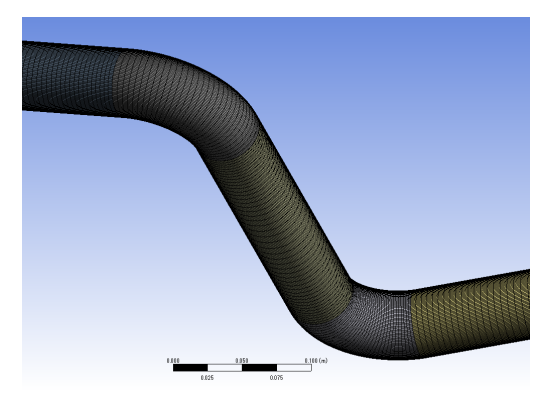

Figure 9. Detail view around the double bent. 


\subsection{Results of Simulation Calculations}

Examples of the numerical calculation are shown Figures 10-15. Velocity vectors at the downstream of $3 D$ from the double bent are shown in Figure 10 in the case of straight case $\left(\alpha=0^{\circ}\right)$.

Apparent twin vortices exist and they are almost the same size. In this straight case $\left(\alpha=0^{\circ}\right)$, the sizes of twin vortices are almost same each other also at other positions as $4 \mathrm{D}, 5 \mathrm{D}$, etc.

Figure 11 depicts a contour of cross-sectional vorticity (vorticity calculated with only cross-sectional velocity component, without velocity component along the pipe direction) at the same condition, $3 D$ and $\alpha=$ $0^{\circ}$. Almost the same size and intensity vortices in case $\alpha=0^{\circ}$ are shown in Figure 11.

When the out-of-plane angle exists (not $\alpha=0^{\circ}$ ), the twin vortices are twisted and deformed. Figure 12 depicts velocity vectors at the downstream of $3 \mathrm{D}$ from the double bent and the flow is bended to right side with the out-of-plane angle, $\alpha=8^{\circ}$. In the case, the twin vortices are twisted and their sizes become different. Cross-sectional vorticity reveals their size and strength more clearly. In Figure 13, the right vortex is stronger than the left one, and the left vortex becomes larger than the right one. Larger $\alpha$ makes this tendency more obvious. Figure 14 and Figure 15 depict velocity vectors and cross-sectional vorticity at $3 D$ in case $\alpha=16^{\circ}$. The right side vortex is clear, however, the left side one is enlarged and becomes unclear.
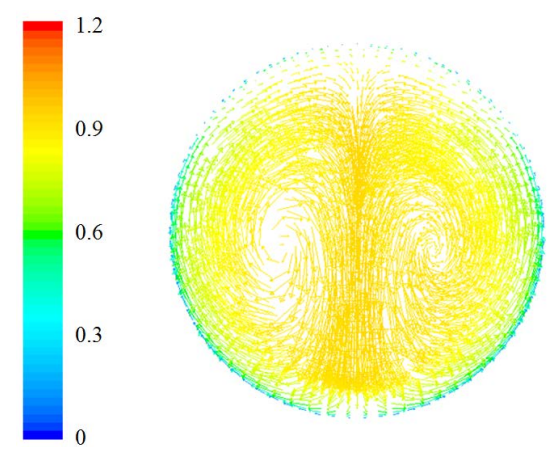

Figure 10. Velocity vectors at cross-section of $3 D$ from double bent with $\alpha=0^{\circ}$.
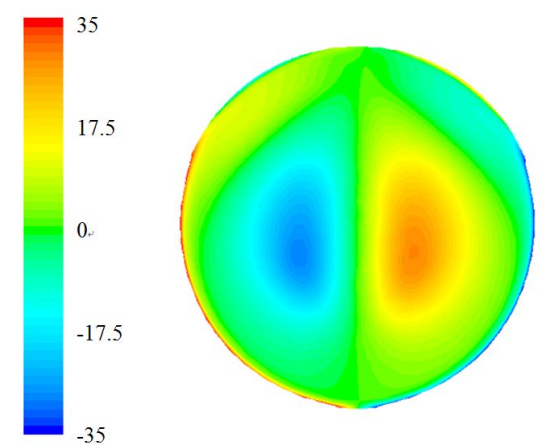

Figure 11. Contour of cross-sectional vorticity at cross-section of $3 \mathrm{D}$ from double bent with $\alpha=0^{\circ}$.

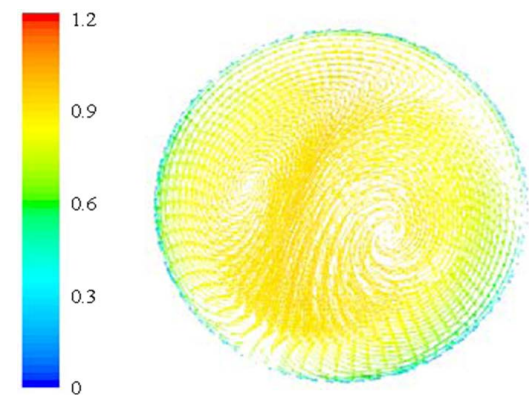

Figure 12. Velocity vectors at cross-section of $3 D$ from double bent with $\alpha=8^{\circ}$. 

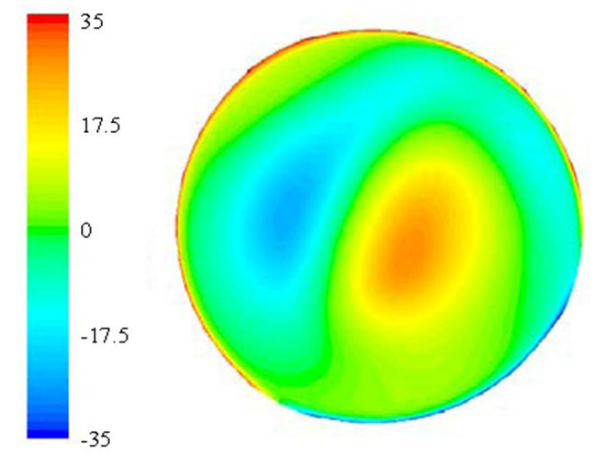

Figure 13. Contour of cross-sectional vorticity at cross-section of $3 \mathrm{D}$ from double bent with $\alpha=8^{\circ}$.
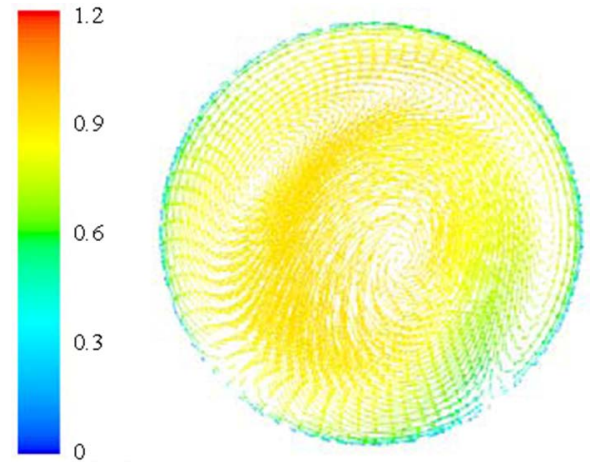

Figure 14. Velocity vectors at cross-section of $3 D$ from double bent with $\alpha=16^{\circ}$.
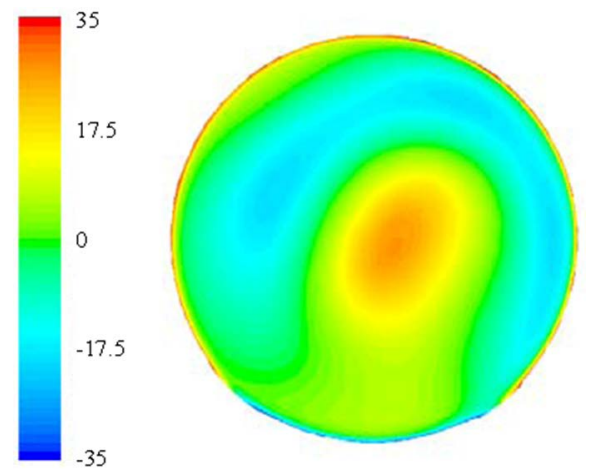

Figure 15. Contour of cross-sectional vorticity at cross-section of $3 \mathrm{D}$ from double bent with $\alpha=16^{\circ}$.

\section{Results Comparison between Experimental and Numerical}

Comparison between these numerical data and experimental data is executed to confirm the accuracy of the calculation results. The experimental data were already obtained, as already described in chapter 2.2, for the distance from double bent as $3 D, 4 D, 5 D$ (and other distances of $12 D$ or $20 D$, as shown in Figure 4) and out-of-plane angle, $\alpha$, as $0^{\circ}, 8^{\circ}$ and $16^{\circ}$ obtained using Ultrasonic Velocity Profiler (UVP).

Simple velocity profiles cannot be compared with UVP results because the UVP profiles have only information of velocity component along with their measurement lines. Therefore, profiles of velocity components along the measurement lines are reproduced by using numerical results. The velocity information along the lines of virtual measurement line was extracted, and the extracted data were converted to profiles of velocity component along the virtual measurement line like as UVP profile. The profiles were compared with experimental UVP data. 
Figures 16(a)-(d) depict the extracted velocity profiles in the case of $\alpha=16^{\circ}$ along the virtual measurement lines, which angle is determined as same as ones of the relating experiment. These profiles indicate large values near the both side of the profiles, which means the flow is faster around the pipe wall. However, numerical result shows a large peak around the center, especially in the results for circumferential angle, $\phi=$ $0^{\circ}-50^{\circ}$ and $200^{\circ}-350^{\circ}$. On the other hand, a peak around the center doesn't apparently exist in the experimental result of Figure 7. These results show that the twin vortices were not so strong in this experimental result than in the numerical results, or numerical simulation overestimated the vorticity of the twin vortices. However, flow behavior around the pipe wall shows better agreement. Figure 17 depicts normalized velocity at the position of $r / R=0.6$ (circle) and $r / R=-0.6$ (triangle) changing circumferential angle. Horizontal axis indicates circumferential angle, $\varphi$, and vertical axis means normalized velocity. Solid marks mean experimental results and open marks mean numerical results. The data of solid and open circle roughly agree each other, and the triangle data also. Therefore, only strength of flow between the twin vortices is overestimated in numerical, and other flow behavior roughly agrees between experimental and numerical results. The total discrepancy between numerical results and experimental is summarized in Table 2. The numerical results suggest that the strength of twin vortices can be changed easily by small experimental factors, wall roughness, wall gap at the pipe connection, and so on.

\section{Conclusion}

Numerical analyses for double bent system which consist of twin vortices have been studied using CFD code FLUENT. Those numerical results were compared with experimental results obtained by UVP, which is a noble measurement method for obtaining instantaneous spatio-temporal velocity profiles. The flow behavior by numerical results and experimental results are compared.

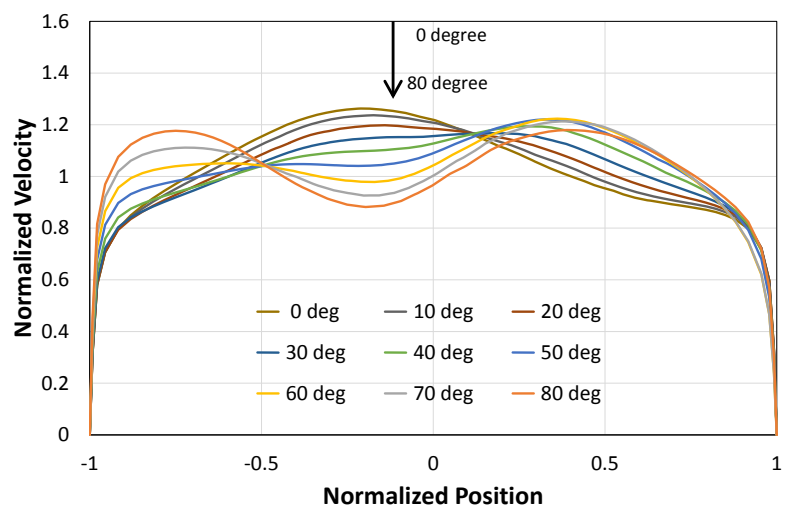

(a)

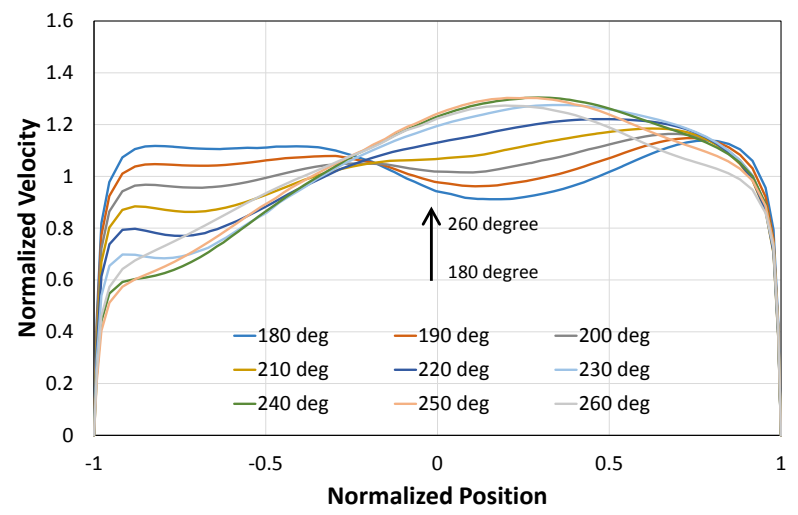

(c)

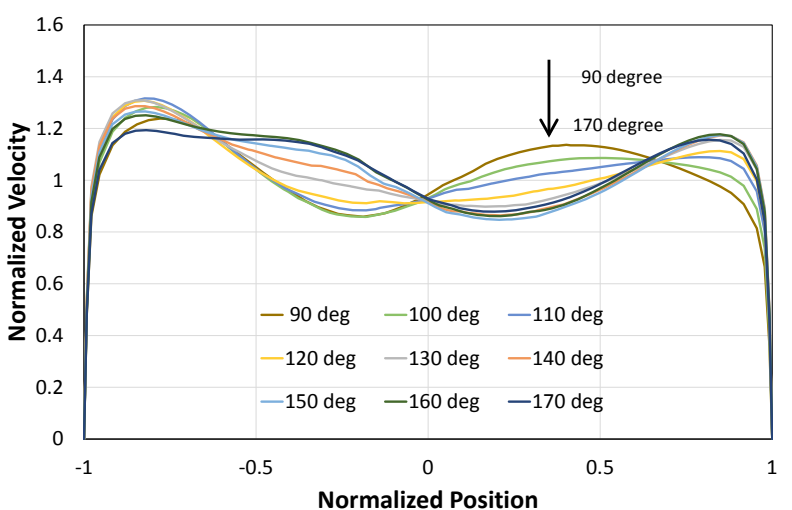

(b)

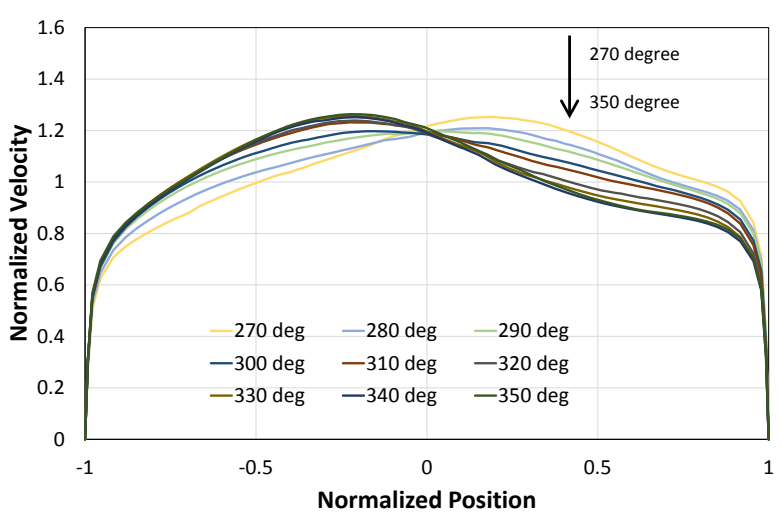

(d)

Figure 16. Normalized radial velocity profiles for every $10^{\circ}$ circumferential angles obtained by CFD code numerically at 3 $\mathrm{D}$ after the double elbow, $\alpha=16^{\circ}$. (a) $\phi=0^{\circ}-80^{\circ}$; (b) $\phi=90^{\circ}-170^{\circ}$; (c) $\phi=180^{\circ}-260^{\circ}$; (d) $\phi=270^{\circ}-350^{\circ}$. 


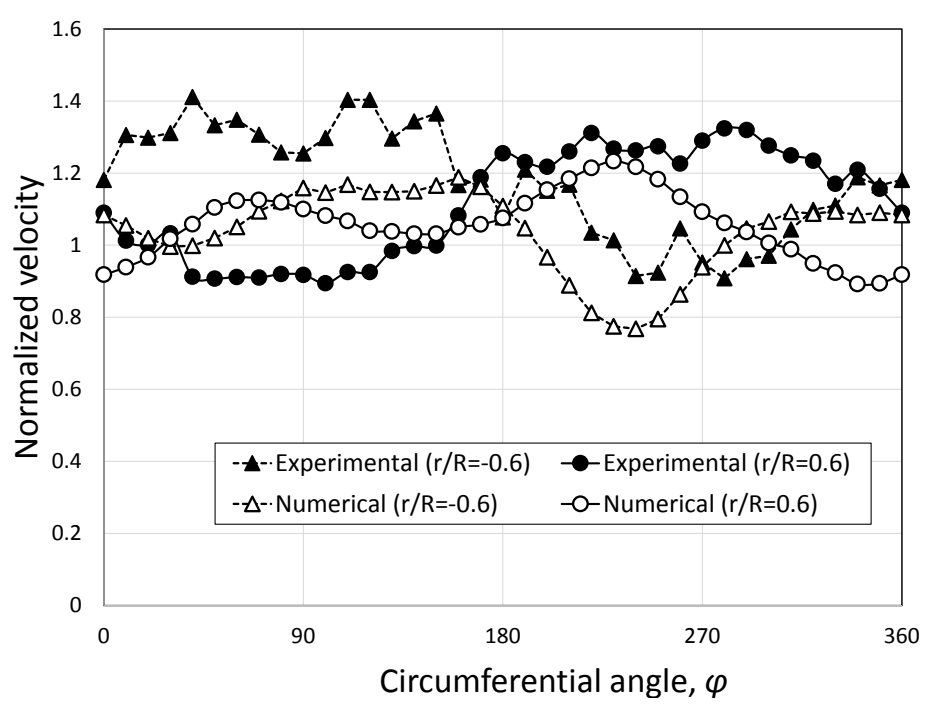

Figure 17. Comparison between experimental result (solid) and numerical result (open) about normalized velocity at $r / R=-0.6$ (triangle) and $r / R=0.6$ (circle) changing circumferential angle, $\varphi$.

Table 2. Error between numerical results and experimental ones as a form of UVP profile.

\begin{tabular}{cccc}
\hline$\alpha$ & $3 D$ & $4 D$ & $5 D$ \\
\hline 0 & $-15.5 \%$ & $-14.0 \%$ & $-16.8 \%$ \\
16 & $-14.0 \%$ & $-19.7 \%$ & $-24.4 \%$ \\
\hline
\end{tabular}

UVP observation is a noble method to obtain an instantaneous profile of velocity distribution, however, the UVP observation is not still enough to understand three-dimensional flow behavior. Therefore, numerical calculation of CFD was utilized to help understanding about complicated flow behavior of double bent pipe system.

The numerical results revealed the details of flow behavior in the double bent system. Behavior of twin vortices, shapes and vorticities are clarified. Out-of-plain angle makes the vortices deformed and the vorticities weaken.

Comparison between numerical and experimental results was carried out by converting numerical results into velocity component profiles like as UVP profiles. The comparison revealed thatnumerical results can predict the characteristic flow behavior of the double bent pipe system, especially near the pipe wall. However, discrepancy between numerical results and experimental results was also found. The discrepancy may cause minor experimental factors like wall roughness, wall gap at the pipe connection, and so on. Study about causes of this discrepancy may develop calculation method for more accurate flow prediction.

\section{References}

[1] Mattingly, G.E. and Yeh, T.T. (1991) Effect of Pipe Elbows and Tube Bundles on Selected Types of Flowmeters. Flow measurement and Instruments, 2, 4-13. http://dx.doi.org/10.1016/0955-5986(91)90050-2

[2] Takeda, Y. (1995) Velocity Profile Measurement by Ultrasonic Doppler Method. Experimental Thermal and Fluid Science, 10, 444-453. http://dx.doi.org/10.1016/0894-1777(94)00124-Q

[3] Takeda, Y., Furuichi, N., Mori, M., Aritomi, M. and Kikura, H. (2000) Development of a New Flow Metering System Using UVP. Preliminary Performance Assessments Using NIST flow Standards. ASME Fluids Engineering Division Summer Meeting.

[4] Mori, M., Takeda, Y., Taishi, T., Furuichi, N., Aritomi, M. and Kikura, H. (2002) Development of a Novel Flow Meter System Using Ultrasonic Velocity Profile Measurement. Experiments in Fluids, 32, 153-160.

[5] Wada, S., Kikura, H., Aritomi, M., Mori, M. and Takeda, Y. (2004) Student Program—Development of Pulse Ultra- 
sonic Doppler Method for Flow Rate Measurement in Power Plant Multilines Flow Rate Measurement on Metal Pipe. Journal of Nuclear Science and Technology, 41, 339-346. http://dx.doi.org/10.1080/18811248.2004.9715493

[6] Treenuson, W., Tsuzuki, N., Kikura, H., Aritomi, M., Wada, S. and Tezuka, K. (2013) Accurate Flowrate Measurement on the Double Bent Pipe Using Ultrasonic Velocity Profile Method. The Japanese Society for Experimental Mechanics, 13, 200-211.

[7] Treenuson, W., Tsuzuki, N., Kikura, H. and Wada, S. (2014) Effect of Out-of-Plane Double Bent Pipe on Ultrasonic Flow Metering. Proceedings of SPIE, to be published.

[8] Yuki, K., Hasegawa, S., Sato, T., Hashizume, H., Aizawa, K. and Yamano H. (2011) Matched Refractive-Index PIV Visualization of Complex Flow Structure in a Three-dimensionally Connected Dual Elbow. Nuclear Engineering and Design, 241, 4544-4550. http://dx.doi.org/10.1016/j.nucengdes.2010.12.026

[9] Yuki, K., Hasegawa, S., Sato, T., Hashizume, H., Aizawa, K. and Yamano, H. (2009) Unsteady Hydraulic Characteristics in Large-diameter Pipings with Elbow for JSFR. 3. Flow Structure in a 3-dimensionally Connected Dual Elbow Simulating Cold-Leg Piping in JSFR. Proceedings of 13th International Topical Meeting on Nuclear Reactor Thermal Hydraulics (NURETH-13).

[10] Takeda, Y. (2012) Ultrasonic Doppler Velocity Profiler for Fluid Flow. Springer, Japan. http://dx.doi.org/10.1007/978-4-431-54026-7

[11] NIST (2010) Standard Reference Database 23: Reference Fluid Thermodynamic and Transport Properties-REFPROP ver 9.0.NIST, US. 
Scientific Research Publishing (SCIRP) is one of the largest Open Access journal publishers. It is currently publishing more than 200 open access, online, peer-reviewed journals covering a wide range of academic disciplines. SCIRP serves the worldwide academic communities and contributes to the progress and application of science with its publication.

Other selected journals from SCIRP are listed as below. Submit your manuscript to us via either submit@scirp.org or Online Submission Portal.
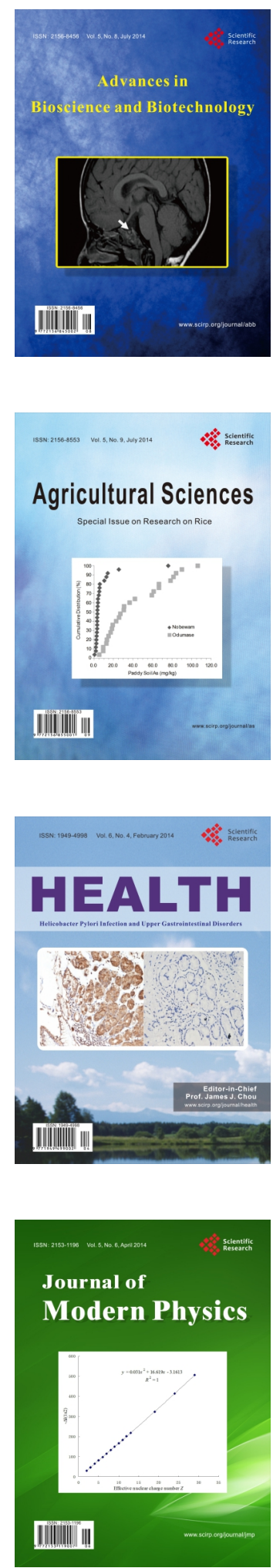
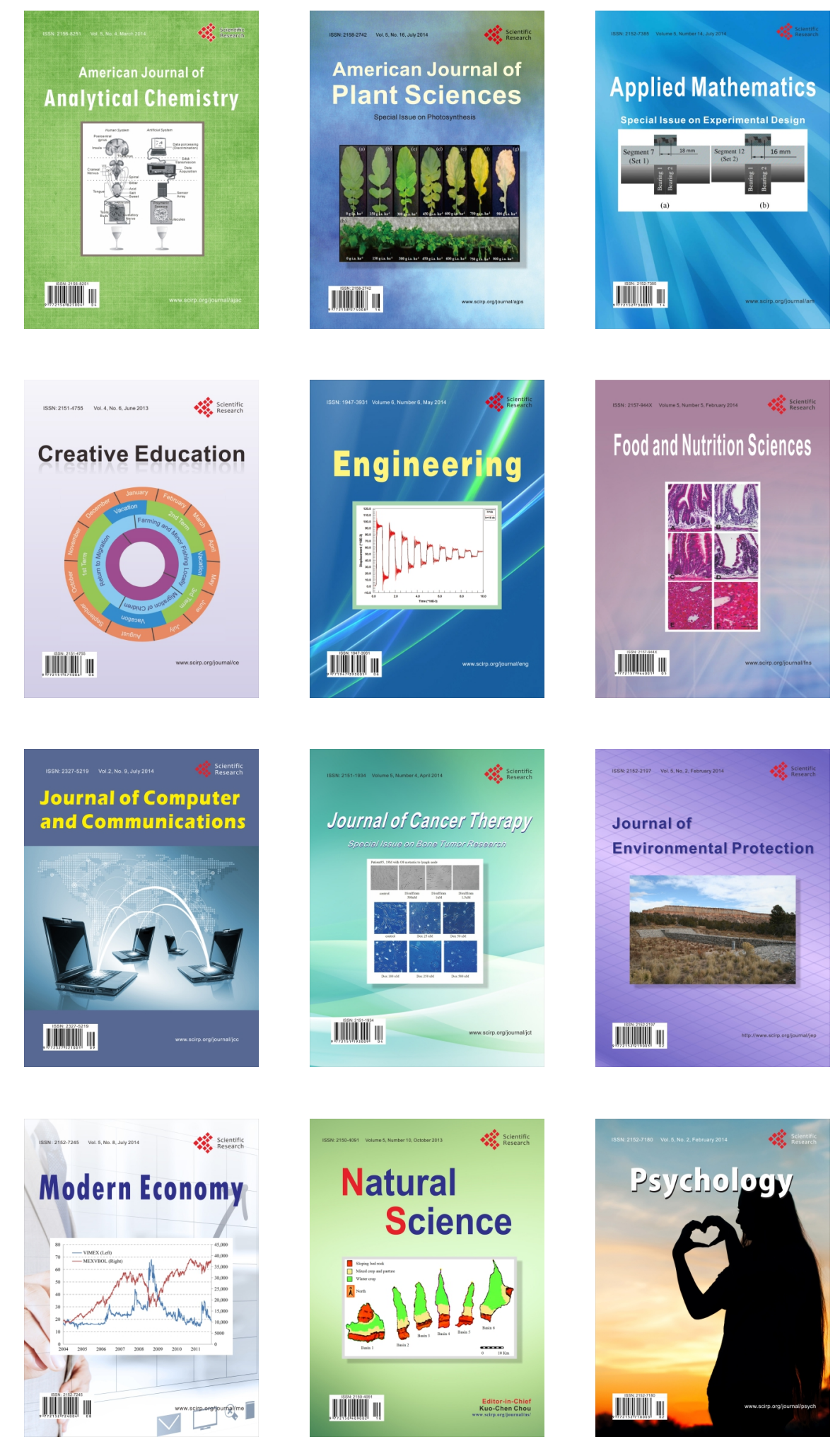